\title{
L'analyse stratégique de la gestion environnementale : un cadre théorique pour penser l'efficacité en matière d'environnement
}

\author{
Laurent Mermet ${ }^{\mathrm{a}}$, Raphaël Billéb ${ }^{\text {, Maya Leroy }}{ }^{\mathrm{c}}$, Jean-Baptiste Narcy ${ }^{\mathrm{d}}$, Xavier Poux ${ }^{\mathrm{e}}$ \\ a Sciences de gestion, ENGREF, 19 avenue du Maine, 75015 Paris, France \\ b Sciences de l'environnement, ENGREF, 19 avenue du Maine, 75015 Paris, France \\ c Sciences de gestion, ENGREF, BP 44494, 34093 Montpellier cedex 5, France \\ d Sciences de l'environnement, Applications des sciences de l'action (AScA), 8 rue Legouvé, 75010 Paris, France \\ e Agro-économiste, AScA, 8 rue Legouvé, 75010 Paris, France
} L'approche interdisciplinaire de l'action en faveur de l'environnement fait débat. Dans cet article, les chercheurs du groupe
de Recherche en gestion sur les territoires et l'environnement (RGTE) présentent les principes qui fondent leur démarche et lui
donnent sa cohérence et son originalité. Ainsi explicités et synthétisés, ceux-ci n'en peuvent que mieux être soumis à la discussion.

La Rédaction

\section{Mots-clés :}

gestion; environnement; stratégie : action; évaluation

\section{Keywords:} management; environment; strategy; policy; evaluation

\begin{abstract}
Résumé - Les exemples abondent de problèmes environnementaux que les efforts collectifs entrepris jusque-là ne suffisent pas à traiter efficacement. La question de la cohérence et de l'efficacité de l'action, au regard des responsabilités environnementales, est donc de plus en plus centrale. Elle appelle de manière urgente des travaux approfondis. Encore faut-il qu'ils puissent s'appuyer sur un cadre théorique adéquat. C'est dans cette perspective que le présent article présente l'analyse stratégique de la gestion environnementale (ASGE). Il en rappelle les origines et les fondements. Il montre en quoi l'ASGE permet de surmonter des contradictions et des impasses omniprésentes dans des champs d'étude et de recherche cruciaux pour l'avenir de l'environnement et du développement durable, comme la gestion intégrée des ressources et des territoires, ou l'évaluation des politiques publiques en matière d'environnement.
\end{abstract}

\begin{abstract}
Strategic Environmental Management Analysis: a Framework for Assessing Effectiveness in the Pursuit of Ecological Goals. Over the last decades, environmental issues have assumed a growing importance on political agendas. Despite the public commitments made (laws, international conventions, political statements) and the varied, ambitious and complex management systems set up (institutions, planning procedures, financial tools...), examples in all environmental areas (biodiversity, water management, air and varied, pollution... ) show that in many situations processes causing serious environmental degradation are out of control. Analysing and assessing the coherence and effectiveness of actions in the light of environmental commitments must more than ever be a central issue for environmental studies. However, developing new in depth analyses of such issues requires an appropriate theoretical framework. This paper aims to show that "Strategic Environmental Management Analysis" (SEMA), a theoretical framework developed to this end over the last twenty years, provides precisely the foundations needed. The paper summarises the framework's background in the 1980s and its foundations in strategic approaches of organizational sociology and strategic management. It shows how the framework, when implemented using appropriate methodologies, can be instrumental in overcoming widespread contradictions, confusions or misconceptions in fields that are crucial for sustainable development and environmental management. In the paper, the theoretical reframing promoted by SEMA is applied in two fields taken as examples: integrated coastal management, and environmental policy evaluation.
\end{abstract}

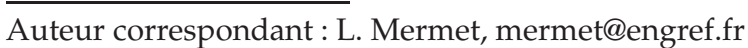


Depuis quatre décennies, l'émergence spectaculaire de la question environnementale sur les scènes politiques et scientifiques a dirigé l'attention des acteurs de la vie publique, et des chercheurs, vers les multiples systèmes écologiques dont les qualités sont menacées. La place que prennent les problèmes de ce type sur les scènes publiques va de pair avec un foisonnement d'initiatives - sociales, politiques, administratives - pour les prendre en charge. Cette activité multiforme a débouché sur deux types d'acquis. D'une part, elle a mené à la constitution progressive d'un ensemble d'engagements pris visà-vis du public en matière de gestion de l'environnement (composé, par exemple, des lois sur la protection de la nature, de la convention internationale de Ramsar sur les zones humides, des protocoles de réduction des émissions de gaz à effet de serre, etc.). D'autre part, elle a mis en place des dispositifs de gestion innombrables, divers, souvent complexes et parfois de grande ampleur (par exemple, les aires protégées, les mesures agrienvironnementales, les taxes parafiscales pour la gestion des déchets, etc.).

Tout ceci n'empêche pas que les processus qui aboutissent à la dégradation des écosystèmes restent pour une bonne part à l'œuvre, voire s'accélèrent : l'efficacité et la cohérence de l'action en matière d'environnement constituent dès lors une question centrale. Il y a là un défi pratique essentiel pour les acteurs de l'environnement. Mais celui-ci se double - c'est le sujet du présent article - d'un défi théorique. Pour «tenir» les engagements environnementaux en pratique il faut aussi, dans les débats et les recherches sur l'environnement, «tenir » les concepts et les raisonnements de la perspective environnementale. C'est bien là le travail de la théorie, qui consiste - nous empruntons ces formulations à Gilles Deleuze - «à développer jusqu'au bout ${ }^{1}$ les implications nécessaires d'une question formulée », à porter les "nouvelles distributions qu'elle impose aux êtres et aux choses ». Défi central, face à certaines forces sociales, économiques ou académiques qui passent aujourd'hui la question environnementale au hachoir de leurs redéfinitions entrecroisées, jusqu'à la priver quasiment de sens.

La perspective de recherche en gestion qui est la nôtre imprime à ce travail théorique nécessaire des attendus spécifiques. D'une part, elle appelle des constructions conceptuelles au sein desquelles les questions d'action, de responsabilité, les liens qui les unissent, occupent une place déterminante ${ }^{2}$. D'autre part, elle débouche sur des travaux où ce qui est mis à l'épreuve avant tout, c'est

\footnotetext{
1 Souligné par G. Deleuze.

2 Dans le rapport de telles recherches à l'action, ce ne sont pas la finalisation, la visée d'action concrète qui sont au centre (elles sont d'ailleurs absentes dans plusieurs des recherches présentées plus loin), mais bien le projet de "penser », en assumant leur complexité et leur ambiguïté concrètes, les problèmes d'action, les questions d'efficacité.
}

la capacité d'un (re)cadrage théorique proposé à fonder des lectures qui renouvellent, d'une façon éclairante aux regard des questions de responsabilité et d'action, la manière dont on peut articuler entre eux les discours gestionnaires qui s'entrecroisent, les dispositifs de gestion qui s'enchevêtrent.

Plus généralement, c'est bien ce travail réorganisateur qui est le moment central de toute entreprise théorique ${ }^{3}$. S'agissant d'action environnementale, et de son efficacité, la préoccupation fondatrice et organisatrice des travaux présentés ici est de fonder, de construire et d'instruire des analyses qui tiennent clairement en regard à la fois (1) un objet écologique clairement défini, (2) les causes de sa dégradation (qui fait problème environnemental), (3) les conditions de l'arrêt de cette dégradation, de sa prévention ou d'une éventuelle restauration et (4) les situations concrètes d'action où se trouvent pris les acteurs sociaux qui s'engagent dans des initiatives pour obtenir des changements qui permettent de résoudre le problème.

$C^{\prime}$ est à cette tâche que le groupe de Recherche en gestion sur les territoires et l'environnement (RGTE) ${ }^{4}$ consacre depuis plusieurs années une partie importante de ses travaux, à travers le développement de l'analyse stratégique de la gestion environnementale (ASGE). Le présent article a pour but de proposer une vue d'ensemble de ce cadre théorique, depuis sa formulation initiale jusqu'à ses déclinaisons actuelles.

Dans un premier temps, nous nous placerons d'un point de vue rétrospectif. Nous rappellerons le contexte et les enjeux de la première formulation de l'ASGE dans les années 1980 ; nous en résumerons les fondements et les spécificités; nous montrerons les phases successives de son développement dans les années 1990 et 2000. Dans un second temps, nous montrerons, en nous appuyant sur des travaux de notre groupe, en quoi le cadrage théorique de l'ASGE permet d'ouvrir de nouvelles voies dans des domaines importants pour l'environnement ${ }^{5}$. Il débouche en particulier sur des propositions théoriques et méthodologiques qui, en permettant une prise de recul et un recadrage, permettent de remédier à certaines contradictions et impasses dont souffrent les approches les plus courantes de la gestion intégrée, du développement durable, de l'évaluation des politiques publiques en matière d'environnement. Dans un troisième temps, nous

\footnotetext{
${ }^{3}$ Quant à la mise en comparaison ou en dialogue avec d'autres propositions théoriques, ce moment (même s'il a son importance) est second et sera ici seulement esquissé. Gageons que le présent texte suscitera des débats qui lui profiteront.

${ }_{4}$ Dont les auteurs du présent article sont membres.

${ }_{5}$ Nous ne pourrons pas, dans le cadre restreint de cet article, développer un exemple en détail ; nous avons essayé, en revanche, de fournir systématiquement au lecteur les indications bibliographiques qui lui permettront d'accéder au corpus de nos travaux, où il trouvera notamment des études de cas approfondies. Les références correspondantes sont marquées d'un losange $(\downarrow)$ dans la liste en fin d'article.
} 
expliciterons les différents niveaux de travail conceptuel que combinent les analyses stratégiques de la gestion environnementale, depuis les problèmes les plus généraux de cadrage théorique jusqu'à la diffusion d'outils méthodologiques. Pour conclure, nous esquisserons des perspectives pour la poursuite de nos travaux et, plus largement, de ceux des chercheurs qui partagent avec nous un intérêt pour l'instruction des questions concernant la mise en cohérence des engagements environnementaux et des dispositifs de gestion.

\section{Fondements de l'ASGE, étapes et état de son développement}

L'analyse stratégique de la gestion environnementale prend ses racines dans nos travaux effectués dès les années 1980 pour formuler, à partir d'études de cas très diverses, une théorie de la pratique de la gestion environnementale (Mermet, 1991 et 1992). Ce travail s'est largement appuyé sur les bases théoriques plus larges du réseau de recherche qui, de 1976 à 1987, a développé la « gestion patrimoniale » (Barouch, 1989; Bertier et de Montgolfier, 1979; Mermet, 1981; de Montgolfier et Natali, 1987; Ollagnon, 1979 et 1987). Il en partageait - et en partage toujours - certains fondements essentiels dont trois doivent ici être soulignés.

D'abord, une vision systémique des situations de gestion qui trouve sa source dans l'analyse systémique largement répandue des années 1970 et 1980 (voir, par exemple, de Rosnay, 1975) puis, de façon plus précise, dans les écrits de Crozier et Friedberg (1977) d'une part, et d'autre part dans ceux de Mintzberg (1989) sur la structure et la dynamique des organisations. Ceci conduit sous des formes diverses à replacer toute situation de gestion environnementale dans une lecture des systèmes d'action où elle s'inscrit et où sont noués ensemble, dans une organisation qu'il s'agit d'élucider, des éléments et des relations multiples, qui ressortent aussi bien d'une qualification sociale (des acteurs, des règles, des enjeux) qu'écologique (des animaux, des plantes, des milieux, etc.).

Ensuite, la visée générale, normative, d'un travail mû par le souci de favoriser une prise de responsabilité croissante de groupes humains à constituer ou à réorganiser, face aux crises, notamment écologiques, qui menacent.

Enfin, une conception, influencée notamment par les systémiciens de l'école de Palo Alto (Barouch, 1989; Watzlawick et al., 1977), par les mouvements de recherche clinique (Berry, 1983; Riveline, 1983), qui placent le travail du chercheur dans tous ses aspects - cadrages théoriques et grilles de lectures, relations au terrain, justification et finalisation des problématiques, etc. - en relation étroite avec la situation de l'intervenant, et demandent au chercheur d'assumer le lien complexe mais insécable qui relie ces deux gestes : comprendre et agir pour transformer.

Cependant, la plupart des approches développées dans le champ de l'environnement sur ces mêmes bases - par exemple, la gestion patrimoniale, la gestion intégrée, le développement durable, etc. - reviennent en dernière analyse à instituer comme système d'action pour remédier à un problème environnemental le même système d'action qui porte en lui, souvent profondément inscrites, les causes de ce problème et l'impuissance ou la réticence à les résoudre. Il y a là une contradiction essentielle, que ces approches entendent lever tant sur le plan théorique que pratique, en posant sous des formes diverses l'hypothèse d'un potentiel de transformation à mobiliser par une meilleure coordination : amélioration de la communication, changement des procédures, ajout de nouvelles institutions de coordination, etc.

Même si ces approches qui posent le problème environnemental en termes de coordination ou d'action collective en sont encore à leurs débuts au milieu des années 1980, la réflexion critique sur leurs fondements, le constat qu'elles méconnaissent ou minorent systématiquement certains aspects des dossiers d'environnement ${ }^{6}$, conduisent à affirmer un désaccord théorique fondateur de notre démarche ${ }^{7}$. Résumons-le en quelques mots : s'il y a un "problème » écologique que la gestion existante peine à résoudre, il faut envisager de changer le système de gestion; l'amélioration de la coordination, la collaboration dans l'action ne sont que des aspects particuliers de ce changement, qu'il n'y a pas de raison de considérer a priori comme centraux, encore moins comme suffisants. La problématique de changement, intrinsèque à la plupart des situations de gestion environnementale rencontrées, nous incitait à mettre au centre de nos analyses le projet d'identifier, dans les situations concrètes de gestion de l'environnement, les conditions d'un changement de gestion, sans les postuler au départ...

D'où la proposition (Mermet, 1991 et 1992) d'un cadre pour une analyse stratégique de la gestion de l'environnement, articulé autour de quatre principes organisateurs.

1. Appuyer l'analyse du système d'action lié à un problème d'environnement sur une définition préalable, en termes écologiques, de l'objet environnemental à prendre en compte et des objectifs poursuivis. La formule un peu provocatrice «buts dans la nature,

\footnotetext{
${ }^{6}$ Par exemple, le rôle essentiel souvent joué par le mouvement environnemental, le poids des résistances au changement, la domination exercée à tous les niveaux de la décision par des réseaux d'intérêts sectoriels, etc.

7 Pour un réexamen récent de ce désaccord, voir Leroy et Mermet (2004).
} 
moyens dans la société » insiste sur la ponctuation ${ }^{8}$ très spécifique qu'opère, dans le continuum socioécologique, l'opération particulière qui consiste à répondre à une question précise de responsabilité sur l'état de l'environnement.

2. Prendre en compte, dans le diagnostic de la gestion de cet objet environnemental, l'ensemble des actions anthropiques qui, consciemment ou non, intentionnellement ou non, ont une influence déterminante sur ses qualités : c'est cet ensemble que l'on définit comme la "gestion effective». Ce concept, souvent mal compris, opère un décalage majeur par rapport aux habitudes qui restreignent en général l'usage du mot gestion à l'intervention délibérée sur une organisation. Pourtant, le concept de gestion désigne bien aussi l'exercice de fait, conscient ou non, d'une responsabilité d'ensemble. Ainsi, une entreprise mal gérée n'est pas tant celle que ses dirigeants «mal-gèrent » consciemment et activement, que celle où le management ne parvient pas à insuffler à la gestion effective la cohérence nécessaire avec les objectifs de gestion.

3. Apporter une attention centrale aux acteurs qui ont pour mission principale de provoquer des changements appropriés de la gestion effective de l'objet écologique : ce sont les "acteurs d'environnement », opérateurs de la «gestion intentionnelle». Ce concept est souvent, lui aussi, saisi avec peine. La tentation est forte, en effet, de considérer comme acteurs d'environnement tous ceux qui sont impliqués dans le problème environnemental que l'on considère, ou bien tous ceux qui mènent des actions à objectif environnemental affiché. Nous lui donnons un sens plus étroit : le ou les acteurs qui, dans une situation donnée, jouent effectivement (à la fois dans le discours et par leurs actions constatées), vis-à-vis des acteurs responsables des processus dommageables pour l'environnement ou des acteurs régulateurs (élus territoriaux, préfet, etc.), un rôle d'agent de changement en faveur de l'objectif environnemental pris en référence. Là encore, ce concept porte le poids d'un déplacement de l'analyse qui fait passer le problème d'environnement du statut de "problème-collectif-à-résoudre-tous-ensemble » à celui de responsabilité collective qui ne sera pas honorée sans l'intervention stratégique efficace $\mathrm{d}^{\prime}$ un agent de changement, en général minoritaire, au moins au départ.

4. Replacer ces analyses dans la perspective dynamique d'un système de gestion qui change et se structure au fil du temps sous l'effet structurant des conflits,

${ }^{8}$ Ce concept essentiel, emprunté aux travaux de l'école de Palo Alto (Watzlawick et al., 1977), désigne le choix par lequel un observateur, devant un enchaînement continu d'événements (causalité circulaire ou enchevêtrée, par exemple), fonde une ligne particulière d'analyse en désignant l'un des moments de l'enchaînement comme premier. par lesquels les préoccupations portées par les interventions de gestion intentionnelle finissent par être partiellement intégrées, ce caractère intégré caractérisant avant tout un résultat de gestion, même s'il peut aussi porter sur l'intervention de gestion elle-même.

Ces quatre principes pris ensemble permettent - on le verra plus loin sur des exemples - un recadrage déterminant pour l'analyse des problèmes environnementaux.

La notion de stratégie occupe, dans ce cadre d'analyse, une place centrale non seulement en référence généalogique aux approches stratégiques de la sociologie des organisations, mais encore davantage au sens où l'action de changement portée par les acteurs d'environnement consiste fondamentalement à déployer des stratégies capables de surmonter les résistances au changement des acteurs du système de gestion effective déjà en place. S'il fallait résumer en une phrase cette perspective, elle revient à considérer les problèmes environnementaux comme des problèmes de changement $d^{\prime}$ un tout - un socio-écosystème correspondant à un état donné de gestion effective - par l'action stratégique d'une de ses parties - un groupe, un réseau, une institution spécialisée, etc.

À partir du début des années $1990^{\circ}$, ce cadre théorique a été mobilisé afin de développer des innovations méthodologiques pour deux types principaux d'applications : d'une part, des interventions de diagnostic et de planification concertée de la gestion environnementale de territoires ${ }^{10}$; d'autre part, des études d'appui à l'évaluation des politiques publiques en matière d'environnement ${ }^{11}$.

Les enseignements de cette mise à l'épreuve concrète du cadre théorique initial ont été repris, en 1998, dans un texte de synthèse, qui en propose notamment une formulation plus complète (Mermet, 1998). De ces compléments, soulignons seulement ici celui qui concerne la responsabilité écologique. Si l'acteur d'environnement est fondé à intervenir comme acteur de changement sur la gestion effective, c'est que, pris ensemble et chacun pour son fait, les acteurs de celle-ci sont comptables de ses effets écologiques devant la partie du public que ces conséquences écologiques préoccupent. On se situe donc dans la perspective d'un "pluralisme des préoccupations ", qui considère abusive l'exigence que la préoccupation environnementale soit partagée par tous, exigence souvent affichée (implicitement ou explicitement), comme

9 Essentiellement dans le cadre du bureau d'études AScA.

10 On peut citer en particulier ici, pour leur ambition et les innovations auxquelles ils ont donné lieu, le plan départemental d'environnement des Côtes-d'Armor (Poux et Mermet, 1998), le plan de gestion du Val de Saône (Cattan et al., 1996), l'étude sur l'avenir de la gestion de la population relictuelle d'ours du Béarn (Poux et al., 1996).

11 L'évaluation des politiques publiques en matière de zones humides (Mermet et al., 1993) constitue ici la référence principale. 
condition de légitimité des revendications sur l'environnement ou comme préalable à l'action environnementale.

Notre position revient donc à défendre une conception stratégique de l'action - le changement du tout passe par l'action de certains - dans le cadre d'un pluralisme substantiel - la responsabilité de tous au regard des préoccupations de certains. Elle propose ainsi une double alternative aux thématiques centrées sur le souci de coordination et aux conceptions procédurales du pluralisme, qui dominent dans les travaux de recherche français des années 1990.

C'est sur cette base qu'a été engagée, depuis 1997, une nouvelle série de travaux ${ }^{12}$ pour pousser plus loin l'étude des fondements théoriques et des applications méthodologiques d'une analyse stratégique de la gestion environnementale. Le défi relevé par ces travaux s'est trouvé d'autant plus important que la même période (1997-2004) voyait la montée en puissance des approches qui posent l'environnement comme problème de coordination, d'action collective, d'apprentissage, aussi bien dans le champ pratique (planification partenariale, concertation, approches patrimoniales, etc.) que dans le champ des travaux en sciences sociales sur l'environnement. C'est donc dans le contexte du développement rapide d'approches dont nous critiquons les fondements mêmes que s'est déroulée la phase de nos travaux qui arrive aujourd'hui à son terme ${ }^{13}$. Ses résultats sont de deux ordres.

D'une part, elle a permis de mettre à l'épreuve la capacité des principes de l'ASGE à opérer, pour l'analyse de systèmes de gestion de l'environnement divers et complexes, un recadrage qui permette de dépasser certaines impasses fondamentales des approches aujourd'hui dominantes. La section suivante de l'article résume les résultats sur ce plan.

D'autre part, elle a conduit à un réexamen et à des développements théoriques et méthodologiques, thème sur lequel nous reviendrons dans la dernière section.

\section{Un cadre pour réexaminer et évaluer les approches de l'action environnementale}

Le champ de l'environnement est marqué, aussi bien en France qu'au niveau international, par le développement puis l'emprise qu'exercent successivement - ou concurremment - des mouvements

\footnotetext{
12 Essentiellement dans le cadre de l'Engref et du fonctionnement du collectif de recherche RGTE.

${ }^{13}$ Avec notamment l'achèvement de trois recherches doctorales (Narcy, 2004; Billé, 2004 ; Leroy, 2004) et l'aboutissement prochain de trois autres (F. Benhammou, A. Emerit, J.-B. Marsat).
}

influents : planification écologique, évaluation des impacts environnementaux, gestion patrimoniale (Babin et al., 1997; Ollagnon, 1987), gestion participative des aires protégées, gestion adaptative (Walters, 1986), gestion intégrée (Margerum, 1999 ; Margerum et Born, 1995), gestion communautaire (Compagnon, 2000), gestion des communaux (Ostrom, 1990), etc. Ces formules mobilisatrices désignent des mouvements de pensée et d'action à la fois. De pensée, car chacun s'attache à développer un appareillage théorique et méthodologique. Pour ne donner qu'un seul exemple, la gestion intégrée des zones côtières (GIZC), notion apparue vers le début des années 1980, a donné lieu à ce jour à la publication de dizaines d'ouvrages de doctrine et de «bonnes pratiques » (guidelines) et fait l'objet de plusieurs conférences internationales annuelles qui rassemblent des milliers de participants. Mouvements d'action également : pour reprendre l'exemple de la GIZC, cette approche fait aujourd'hui référence pour la prise en charge des problèmes environnementaux du littoral au plan international, et c'est autour de ses guidelines (voir en particulier Cicin-Sain et Knecht, 1998), des discours et des dispositifs qu'elles promeuvent que s'organisent l'action publique internationale, les financements internationaux et, in fine, l'action publique nationale de nombreux pays pour la gestion de leur littoral (Billé, 2004; Kalaora et Charles, 2000).

Chacune de ces approches se présente au départ comme une prise de recul théorique par rapport à celles qui lui préexistent. Mais, une fois qu'elle est devenue à son tour un mouvement académico-politicoadministratif profondément engagé dans ses propres intérêts d'action, sur quel cadre s'appuyer pour évaluer (et donc critiquer) ses réalisations et réexaminer ses fondements et son outillage? La question se pose aujourd'hui avec plus d'acuité que jamais, étant donné l'écart entre l'accumulation des discours, des dispositifs, des approches (et la difficulté d'y effectuer un tri) et la dégradation continue que l'on constate dans de nombreux dossiers d'environnement. L'ASGE offre un cadre utile pour, justement, prendre le champ nécessaire. Nous en donnerons ici deux exemples, portant respectivement sur la gestion intégrée et sur l'évaluation des politiques environnementales.

\section{Gestion intégrée, ou processus d'intégration de l'environnement dans la gestion des territoires?}

La «gestion intégrée des territoires et de l'environnement » repose sur l'idée d'intégrer - ou de faire intégrer - dans les processus et résultats du développement le maintien ou le rétablissement de certaines qualités des systèmes écologiques. Ce concept organisateur 
a été appliqué à la gestion de divers types de milieux ou de territoires : bassins versants, zones humides, littoraux, etc. Mais sa concrétisation sous la forme d'un modèle d'organisation, voire d'un programme d'action, peut donner lieu à des dérives qui, in fine, neutralisent ou contrecarrent le traitement des problèmes environnementaux qui constitue pourtant la base de sa légitimité.

L'étude détaillée des difficultés rencontrées par la mise en œuvre de la gestion intégrée des zones côtières (GIZC), aussi bien dans des pays du Sud qu'en France, montre bien ce phénomène. Ainsi à Madagascar, le plan national de gestion environnemental a mis en place, à côté d'autres unités chargées d'aspects divers de l'action de développement, une unité de gestion intégrée $\mathrm{du}$ littoral. Mais, comme il n'existe pas dans le dispositif d'ensemble une unité dédiée spécifiquement à la prise en charge des problèmes environnementaux, l'unité de gestion intégrée est placée devant un choix insoluble. Soit elle se substitue aux acteurs d'environnement défaillants comme interlocuteur environnemental face aux autres services de développement - mais, dans ce cas, devenue " partie et médiateur », elle tend à se disqualifier comme acteur médiateur, intégrateur des préoccupations environnementales et des autres enjeux du développement. Soit elle se place dans cette position de médiation - mais, dans ce cas, le processus d'intégration de l'environnement ne fonctionne pas, faute d'un interlocuteur qui soit pleinement en position de défendre les points de vue environnementaux face aux autres intérêts sectoriels, au sein des processus d'arbitrage. Finalement, on observe un processus de «sectorialisation de la gestion intégrée » : au lieu d'une coordination globale des projets et programmes de développements sectoriels divers, les actions de gestion intégrée deviennent des projets et programmes parmi les autres. Pour justifier leur existence, ils sont conduits à délaisser toute ambition de gestion globale, pour se trouver une niche d'intervention dans l'un des secteurs délaissés par les programmes préexistants et accessibles aux moyens d'intervention souvent limités de ce nouveau venu qu'est la gestion intégrée (Billé et Mermet, 2002).

Dans l'analyse de cette situation, le cadre théorique de l'ASGE offre un fil conducteur dans la mesure où il dénoue d'emblée la confusion centrale où s'alimentent les risques de dérives inhérents à la gestion intégrée. Pour reprendre les concepts introduits plus haut : en matière de gestion intégrée (a), l'action stratégique de changement (intervention de "gestion intentionnelle ») (b) vise à transformer une "gestion effective " actuelle non intégrée (sectorisée, ignorant l'environnement, etc.) (c) en impulsant un «processus d'intégration » (d) qui doit faire émerger une gestion effective transformée quel'on puisse qualifier d'intégrée. Dès lors que l'on confond dans une notion indifférenciée de gestion intégrée plusieurs de ces moments différents ${ }^{14}$ d'un processus d'intégration toujours en marche, on prend le risque d'enferrer l'analyse et l'action dans des impasses dont nos études de terrain montrent la fréquence, la profondeur et la diversité, au Nord comme au Sud (Billé, 2004).

\section{Évaluation environnementale des politiques publiques}

C'est sur cette même capacité à distinguer et à mettre en rapport, tout au long de l'analyse, les buts de l'action environnementale, les systèmes qu'elle entreprend de changer, les processus dans lesquels elle s'inscrit ${ }^{15}$, que repose l'intérêt de l'ASGE pour l'évaluation des politiques publiques dans le domaine de l'environnement. L'étude réalisée en 1994, en appui au travail de l'instance d'évaluation sur les politiques publiques en matière de zones humides, peut être donnée en exemple ${ }^{16}$. D'emblée, il apparaissait clairement que les deux principales approches consacrées dans le champ de l'évaluation des politiques publiques ne pouvaient convenir dans ce cas.

L'une, la plus classique, mise en avant par exemple par le Conseil supérieur d'évaluation (CSE, 1996), consiste à mettre en regard les objectifs assignés à la politique à évaluer avec ses effets propres. S'agissant d'une politique environnementale, il va de soi que les objectifs environnementaux - ici, la gestion durable des zones humides - sont à placer au fondement de l'évaluation. L'évolution de l'état des systèmes écologiques visés est alors un critère incontournable de l'évaluation. Or, cet état ne dépend pas de manière univoque des actions de protection (la «gestion intentionnelle»), mais bien de tout un ensemble d'actions (la "gestion effective») qui affectent, positivement ou négativement, l'état de ces systèmes. En matière de zones humides, par exemple, l'étude a rapidement montré que des actions publiques (agricoles, forestières, urbaines, etc.) étaient à l'origine

\footnotetext{
${ }^{14}$ Et en particulier, le mode d'intervention et le résultat souhaité.

15 Par cette formulation, nous voulons souligner (1) que l'action environnementale doit souvent viser à provoquer des changements dans les structures (sociales, économiques, etc.), mais que (2) ces structures ne sont pas accessibles directement aux interventions de changement : celles-ci doivent (si l'on rejette comme nous les positions «globalitaires») et ne peuvent que (si l'on adopte comme nous une position "réaliste», stratégique, sur l'action) s'inscrire dans les processus en cours, en y déployant des stratégies susceptibles d'induire des changements futurs dans les structures - et, par là, dans les processus à venir. 16 On pourra se référer notamment aux deux rapports d'étude réalisés par AScA pour l'instance d'évaluation présidée par le préfet Bernard (Mermet et al., 1993; Cattan et Mermet, 1994), au rapport de celle-ci (CIME, 1994) et à des textes de réflexion ou d'évaluation publiés depuis sur ce travail (Mermet, 1996 et 1998; Lascoumes et Setbon, 1996; Bazin et Mermet, 1999; Mermet et Barnaud, 1999).
} 
des plus importants processus dommageables aux zones humides. Pour rendre compte de l'exercice de la responsabilité des pouvoirs publics sur les zones humides, c'est donc l'ensemble des effets de l'action publique sur les zones humides qu'il fallait analyser, et c'est seulement dans ce cadre plus large que l'évaluation des mesures ayant pour but spécifique la protection des zones humides a pu prendre son sens.

La seconde approche courante dans le domaine de l'évaluation des politiques est celle qualifiée de pluraliste (Monnier, 1992). Elle consiste schématiquement à fonder l'évaluation d'une politique publique sur sa mise en discussion par un collectif constitué des acteurs concernés par elle et que l'on a réunis "autour d'une table». Lorsqu'il s'agit d'une politique environnementale dont le but est d'obtenir la transformation d'actions publiques et privées portées par des administrations et des filières économiques et socioprofessionnelles très puissantes (agriculture, transport routier, etc.), cela tend à instaurer un dispositif au sein duquel les opérateurs préoccupés de l'efficacité du changement sont structurellement mis en forte minorité face aux acteurs vis-à-vis desquels ils doivent promouvoir le changement. On se trouve alors dans la situation que nous avons critiquée plus haut : celle où le système d'action même qui provoque le problème environnemental et résiste au changement est chargé d'évaluer les actions entreprises par les pouvoirs publics pour le faire changer. .

Pour échapper à une telle impasse, il est capital (1) de construire l'évaluation de l'action publique en distinguant dès le départ le système de "gestion effective » (l'ensemble des actions publiques qui ont un effet sur le problème d'environnement) et le système de "gestion intentionnelle» (les actions publiques indépendantes des filières responsables des impacts négatifs, et qui ont pour mission d'induire des changements), (2) d'étudier chacun de ces deux systèmes de manière séparée, puis (3) d'analyser leurs interactions de manière à pouvoir déboucher sur une évaluation de la capacité de l'ensemble de l'action publique à faire émerger une gestion efficace du problème environnemental concerné. Faute de pouvoir mettre en regard, d'un côté, les efforts entrepris en faveur des changements nécessaires à l'efficacité environnementale et, de l'autre, les résistances que le système de gestion effective oppose à ces efforts, il n'est pas possible d'apprécier dans quelle mesure une politique environnementale est adéquate ou non.

C'est en s'ancrant dans ces principes et en y adossant les innovations méthodologiques appelées par les caractéristiques spécifiques de l'objet traité, que l'évaluation «zones humides » de 1994 a permis de mesurer clairement les moyens mis en œuvre pour promouvoir, en France, la gestion durable des zones humides, de constater qu'ils ne suffisaient pas ${ }^{17}$ (au moment de l'évaluation) à enrayer la dégradation observée de la situation en la matière et de pointer de façon précise les différentes actions publiques (agricoles, d'équipement, etc.) responsables de cette évolution. C'est sur cette base qu'ont pu s'appuyer une étude des marges de manœuvre envisageables pour améliorer la situation, puis la définition d'un plan national d'action. La mise en œuvre de celui-ci se poursuit encore aujourd'hui (Barnaud et al., 2004), suites qui font désormais qualifier cette évaluation de réussite, alors que dans un premier temps elle a, par les innovations de cadrage sur lesquelles elle reposait, essuyé une appréciation négative du Conseil scientifique de l'évaluation. D'autres recherches plus récentes portant sur des problèmes d'évaluation nous ont permis de mettre à l'épreuve cette capacité du cadre théorique ASGE à fournir une structuration spécifique des questions, suffisamment claire et solide pour servir de point d'appui au travail d'analyse considérable qu'il faut accomplir pour dénouer l'entrelacs des responsabilités - et des dénis de responsabilités - qui caractérise les programmes ou les dispositifs de gestion de l'environnement ${ }^{18}$.

\section{Un travail de (re)cadrage, de mobilisation et d'innovation, qui conduit à développer un corpus théorique et méthodologique}

Si la perspective centrale qu'offre l'ASGE sort confortée de quinze années de travaux, il s'avère aussi indispensable de mieux caractériser les différents types d'activités

\footnotetext{
17 Alors même qu'ils engageaient à peu près la moitié des moyens (humains et financiers) de l'action publique en matière de protection de la nature.

18 Le cadre limité du présent article ne nous permet pas de présenter d'exemple de l'analyse stratégique d'un cas de terrain où est en jeu l'évaluation de la gestion d'un écosystème donné. Nous renvoyons pour cela le lecteur au travail de Maya Leroy (2004) sur la gestion des écosystèmes de la vallée du fleuve Sénégal, le plus abouti des travaux de ce type réalisés jusqu'ici dans notre groupe de recherche. Dans une situation marquée par la multiplicité des dispositifs d'action, qui rendait très difficile toute évaluation, ce travail de réexamen critique a permis de rendre plus lisibles les relations entre les engagements environnementaux (objectifs de référence), l'ensemble des actions publiques qui constituent la gestion effective des écosystèmes du fleuve Sénégal, les dispositifs de gestion environnementale (gestion intentionnelle) et les connaissances sur les processus et les causes de la dégradation des écosystèmes. On y trouvera en outre une discussion théorique approfondie des enjeux de l'adoption d'une perspective d'ASGE pour un tel terrain. D'autres travaux fourniront des exemples en France, à une échelle plus locale : notamment l'analyse des apports et des limites du dispositif de "gestion patrimoniale » affiché dans le dossier de l'ours des Pyrénées (Mermet, 2001), ainsi que les cas de terrain sur la gestion intégrée du littoral dans la recherche de Raphaël Billé (2004).
} 
auxquelles elle donne lieu. Cette explicitation est nécessaire pour éviter le risque d'un malentendu majeur : celui qui consisterait à considérer l'ASGE comme une boîte à outils méthodologique intégrée, qu'il suffirait d'appliquer à un terrain, quel qu'il soit, pour obtenir un résultat garanti d'avance - et labellisé! Il importe tout particulièrement de bien distinguer les niveaux où se déroule un travail d'étude, ou de recherche, en tension entre un travail conceptuel et l'implication dans des dossiers, ou des terrains - souci que partagent avec nous d'autres chercheurs, notamment en sciences de gestion (voir en particulier David, 2000).

À un premier niveau, l'ASGE travaille à une base théorique dans la mesure où elle repose sur l'identification a priori d'un certain nombre de conditions d'ordre très général que doit remplir l'analyse d'une situation, lorsque cette analyse a pour but de répondre à une question de responsabilité et/ou d'action portant sur l'état d'un système écologique. Pris ensemble, ces choix de cadrage fondamentaux (en anglais, on parlerait de framework) sont générateurs d'une position, d'un regard particulier sur les situations à analyser, et structurent une problématique ${ }^{19}$. Reformulons-en les principes directeurs :

1. L'analyste donne à l'état d'un système écologique le statut d'un référentiel externe, fondateur pour l'analyse et l'évaluation d'un système socio-écologique (et pas seulement le statut d'une variable interne dans le fonctionnement de celui-ci).

2. L'ensemble des déterminants de cet état constitue par définition un système socio-écologique que l'on peut analyser sous l'angle à la fois de la responsabilité et de l'action stratégique de changement.

3. L'analyste élabore une lecture de ce système qui prend en compte aussi bien les facteurs bio-physicochimiques que sociaux, les deux restant articulés de façon à conserver la lisibilité des «ponctuations » qui clivent ces deux domaines.

4. Tout au long de ce travail d'interprétation, les multiples choix théoriques, méthodologiques, pratiques, rédactionnels, sont guidés par un double souci : mettre en évidence les responsabilités sur l'état du système (voir 1); produire des interprétations qui aient un sens pour un acteur de référence qui, placé au cœur du système en cause, aurait pour but $d^{\prime} y$ intervenir comme acteur stratégique de changement en faveur de l'environnement.

\footnotetext{
${ }^{19}$ Comme l'écrivent Ostrom et al. (1994), « un cadre théorique oriente l'analyste vers des questions particulières à poser ». Parmi les travaux de notre groupe qui relèvent directement de ce type de recadrage, on peut encore donner comme exemple les propositions de Mermet et Poux (2000) et de Poux (2001) pour une reformulation (à des échelles différentes) de la problématique de l'évaluation des impacts environnementaux de l'agriculture.
}

À ce premier niveau, où le travail théorique est un travail de structuration et d'élaboration des questions environnementales, les recherches sur l'ASGE consistent en une réflexion sur les concepts et principes organisateurs, en tentatives de réélaboration et de reformulation, en discussions avec d'autres perspectives théoriques, qui structurent différemment les questions environnementales ${ }^{20}$.

À un second niveau, il s'agit de traiter ces questions organisatrices. Chacune soulève de multiples questions secondes. Comment mesurer l'état de l'objet environnemental pris en référence? Quelle analyse conduire sur les dynamiques organisationnelles sous-jacentes à une activité économique donnée, génératrice d'impacts négatifs sur l'environnement? Comment mettre en rapport les rhétoriques présentées par les acteurs dans l'espace public et l'analyse de leurs actions? Etc. Pour instruire ces questions, étant donnés la complexité et le caractère multidimensionnel des situations de gestion environnementale, on peut - il faut - mobiliser des ressources théoriques spécifiques. Le plus souvent, elles sont à choisir dans l'immense corpus généré par les différentes disciplines qui peuvent concerner l'environnement. Leur utilisation requiert alors des adaptions plus ou moins profondes. Dans d'autres cas, il faudra innover en faisant des propositions nouvelles. Le cadre théorique de l'ASGE ne peut pas ici répondre directement. Il « fournit un langage métathéorique pour réfléchir à diverses théories du point de vue de leur utilité pour traiter les questions qui importent pour l'analyste » (Ostrom et al., 1994). Il organise un espace de travail ouvert dans lequel rien n'est jamais joué d'avance. Cependant, à mesure que se multiplient les travaux conduits dans la perspective de l'ASGE, viennent s'accumuler des ressources et des développements théoriques - concepts, grilles de lecture, lignes de raisonnement, etc. - en partie importés et adaptés, en partie spécifiques. À ce second niveau, les recherches en ASGE se présentent donc comme l'accumulation progressive et la discussion d'un corpus en développement de théories propres et d'éléments théoriques repris à d'autres perspectives et retravaillés dans une direction spécifique ${ }^{21}$.

\footnotetext{
${ }^{20}$ Sans développer ce thème ici, mentionnons simplement que de telles approches ont été engagées, par exemple autour de la théorie de la justification de Boltanski et Thévenot (1991) (voir Narcy, 2004; Narcy et Mermet, 2003), de la théorie des "politiques de la nature » proposée par Latour (1999) (voir Mermet, à paraître (a)), ou d'approches critiques comme celle proposée par Boltanski et Chiapello (1999) (voir Leroy, 2004 ; Mermet, à paraître (b)).

${ }^{21}$ Il faut citer ici les travaux qui s'attachent à développer des aspects particuliers de l'action environnementale, aspects dont l'analyse stratégique a conduit à mettre en relief l'importance et à orienter le traitement ; par exemple : les résistances anti-environnementales (Benhammou et Mermet, 2003), les stratégies des ONG de protection de la nature (Gaudefroy de Mombynes et Mermet, 2003).
} 
Si on descend encore, à un troisième niveau, vers telle intervention spécifique d'étude ou de recherche, conduire une analyse stratégique de la gestion environnementale suppose que l'on définisse une marche à suivre pour aborder une situation-problème et que l'on choisisse ou que l'on invente des outils méthodologiques à mobiliser à différentes étapes de cette démarche. Le travail d'ASGE effectué à ce niveau suppose de concevoir et de mettre en œuvre des méthodes, des savoir-faire spécifiques pour aborder telle ou telle question et tel ou tel cas de terrain. Il peut donner lieu à deux types différents de développements. D'un côté, il conduit à envisager de proposer à l'avenir des méthodologies stabilisées et reproductibles pour des gammes bien définies de situations similaires, par exemple une méthodologie pour le diagnostic d'un problème environnemental à l'échelle d'un territoire ${ }^{22}$. De l'autre côté, parce qu'aucune méthode standardisée ne permettra d'embrasser l'ensemble des situations (dans le temps, dans l'espace, dans les types de contextes, dans les types de problèmes), il appelle des travaux d'innovation méthodologique destinés à répondre à des situations uniques ou, du moins, nouvelles. Enfin, la conduite d'analyses stratégiques de systèmes de gestion environnementale passe par l'élaboration d'une pratique de plus en plus précise et explicite du terrain, où les questions d'engagement ou non du chercheur, de rapport entre la recherche et les préconisations qui en sont attendues, des liens entre les intérêts des acteurs du terrain et l'intéressement du chercheur, soient traités avec l'attention que ces questions fondamentales méritent (Barbier, 1998; David et al., 2000).

L'enjeu d'une démarche d'analyse stratégique de la gestion environnementale ne peut donc pas être simplement d'appliquer (ou pas) une grille de lecture canonique. Il se situe au contraire dans la présence toujours active des trois niveaux de travail conceptuel où se construit l'ASGE : (re)formulation de la perspective, mobilisation/développement d'un corpus théorique, mise en œuvre et enrichissement d'un répertoire de ressources méthodologiques.

En distinguant et en articulant clairement ces trois niveaux, l'ASGE se dote à la fois d'une cohérence d'ensemble et d'une capacité à évoluer, en bénéficiant notamment des développements continus d'autres recherches, dont elle mobilise - ou critique - les outils et résultats pour répondre à ses propres questionnements.

\section{Conclusion et perspectives}

«Qui peut agir, et comment, pour préserver ou rétablir telle qualité désirable de tel écosystème? » Cette

\footnotetext{
${ }^{22}$ Un peu comme l'approche "systèmes agraires » (Deffontaines et Lardon, 1994), qui se traduit notamment (mais pas seulement) par une méthode standardisée pour le diagnostic de système agraire à l'échelle d'un terroir.
}

question est celle de l'action, de la responsabilité, de l'efficacité environnementales. Ce questionnement trouve notamment sa légitimité (1) dans les attentes des citoyens et des mouvements sociaux qui soulèvent ces questions de responsabilité et d'action environnementale, qui attendent une efficacité concrète, lisible dans l'état des écosystèmes, et (2) dans les engagements officiels pris par les pouvoirs publics, depuis la sphère locale jusqu'aux conventions internationales, pour répondre à cette attente.

Traiter sérieusement ces questions, au sujet d'un problème environnemental donné, c'est ouvrir un dossier complexe, à la fois social, écologique, technique, économique, etc. ; c'est choisir des concepts organisateurs appropriés pour guider une lecture spécifique de ce dossier ; c'est mobiliser, dans la construction de cette lecture, des concepts, des éléments d'analyse, des données, empruntés à diverses disciplines des sciences de la nature, de l'univers, de l'homme et de la société. C'est à de tels travaux de "mise en lisibilité » que nous appelons et que nous voulons contribuer par les cadrages théoriques de l'ASGE et le corpus ouvert de réalisations et de méthodes qui se constitue avec ses développements et ses utilisations.

La situation actuelle du domaine de l'environnement nous donne à penser que cette perspective répondra à un besoin majeur des années qui viennent. En effet, depuis sa genèse au milieu des années 1980, 1'ASGE porte une interpellation critique des approches qui choisissent, sous des formes diverses, de voir les problèmes d'environnement essentiellement (voire même seulement) comme problèmes de coordination entre acteurs (action collective, apprentissage collectif, gestion intégrée et concertée, etc.). Innovantes dans les années 1980, en pleine expansion dans les années 1990, ces approches sont aujourd'hui dans une position d'hégémonie. Sur le plan pratique, la mise en œuvre de dispositifs de gestion fondés sur elles s'est très rapidement généralisée ces dernières années, souvent au détriment d'autres formes d'action tout aussi nécessaires. C'est seulement aujourd'hui que l'on commence à découvrir les limites de ces dispositifs, la fréquence et les conséquences de carences dans leur conception ou leur mise en pratique $\mathrm{e}^{23}$. Leur examen critique, et celui des conceptions sur lesquelles ils reposent, la réflexion sur les moyens de dépasser leurs insuffisances, sont des tâches qui incombent aux chercheurs du champ de l'environnement (en particulier dans les disciplines de sciences sociales) et dont l'urgence ne devrait que croître dans les prochaines années.

\footnotetext{
${ }^{23}$ Pour ne citer qu'un exemple, les travaux de Busca et Salles (à paraître) démontent avec précision les processus par lesquels la mise en œuvre négociée de certaines mesures agrienvironnementales conduit, in fine, à les vider de leur contenu.
} 


\section{Références}

Babin, D., Bertrand, A., Weber, J., et al., 1997. Médiation patrimoniale et gestion subsidiaire, Montpellier, Antananarivo, CiradGREEN.

Barbier, M., 1998. Pratiques de recherche et invention d'une situation de gestion d'un risque de nuisance : d'une étude de cas à une recherche intervention. Thèse de doctorat, Université Lyon 3.

Barnaud, G., Barre, V., Weng, P., 2004. Programme national de recherche sur les zones humides : les actes du colloque de Toulouse (22-24 octobre 2001), Toulouse, BRGM-Agences de l'Eau, MEDD-MAAPAR-METLTM.

Barouch, G., 1989. La Décision en miettes : systèmes de pensée et d'action à l'cuvre dans la gestion des milieux naturels en France, Paris, L'Harmattan.

Bazin, P., Mermet, L., 1999. L'évaluation des politiques « zones humides » de 1994 : son origine, son déroulement, ses résultats, Annales des Mines/Responsabilité E Environnement, 14, 79-89.

- Benhammou, F., Mermet, L., 2003. Stratégie et géopolitique de l'opposition à la conservation de la nature : le cas de l'ours des Pyrénées, Natures Sciences Sociétés, 11, 4, 381-394.

Berry, M., 1983. Une technologie invisible, Paris, Centre de recherche en gestion de l'École polytechnique.

Bertier, P., Montgolfier, J. de, 1979. Tentative pour poser le problème forestier en termes de patrimoine, in Congrès de l'association des ruralistes français.

- Billé, R., 2004. La Gestion intégrée se décrète-t-elle? Une analyse stratégique de la mise en ouvre, entre approche programme et cadre normatif. Thèse de doctorat, Engref, Paris.

- Billé, R., Mermet, L., 2002. Sectoralization of an Integrated Coastal Management Program: A case study in Madagascar, Journal of Environmental Planning and Management, 45, 6, 913926.

Boltanski, L., Chiapello, E., 1999. Le Nouvel Esprit du capitalisme, Paris, Gallimard.

Boltanski, L., Thévenot, L., 1991. De la justification. Les économies de la grandeur, Paris, Gallimard.

Busca, D., Salles, D., à paraître. Quelle performance des dispositifs agri-environnementaux?, in Concertation, décision et environnement : regards croisés, vol. IV, Paris, La Documentation française

- Cattan, A., Dubien, I., Laurans, Y., Mermet, L., 1996. Élaboration d'un plan d'utilisation de l'espace inondable et d'un plan de gestion pour le Val de Saône, Paris, AScA/Syndicat mixte d'études pour l'aménagement du bassin de la Saône et du Doubs.

$\checkmark$ Cattan, A., Mermet, L., 1994. Évaluation des politiques publiques en matière de zones humides : rapport de deuxième phase et recoupement avec la première phase, Paris, AScA/Commissariat général du plan - Instance d'évaluation des politiques publiques en matière de zones humides.

Cicin-Sain, B., Knecht, R.W., 1998. Integrated Coastal and Ocean Management, Concepts and Practises, Washington (D.C.), Island Press.

CIME, 1994. Les Zones humides : rapport de l'Instance d'évaluation, Paris, La Documentation française.

Compagnon, D., 2000. Présentation. Impératifs et contraintes de la gestion communautaire, in Compagnon, D., Constantin, F. (Eds), Administrer l'environnement en Afrique, Kartala/IFRA.

Crozier, M., Friedberg, E., 1977. L'Acteur et le Système, Paris, Le Seuil.
CSE, 1996. Petit guide d'évaluation des politiques publiques, Paris, La Documentation Française.

David, A., 2000. Logique, épistémologie et méthodologie en sciences de gestion : trois hypothèses revisitées, in David, A., Hatchuel, A., Laufer, R. (Eds), Les Nouvelles Fondations des sciences de gestion : éléments d'épistémologie de la recherche en management, Paris, Vuibert, 83-109.

David, A., Hatchuel, A., Laufer, R. (Eds), 2000. Les Nouvelles Fondations des sciences de gestion : éléments d'épistémologie de la recherche en management, Paris, Vuibert.

Deffontaines, J.-P., Lardon, S. (Eds), 1994. Itinéraires cartographiques et développement, Paris, INRA Éditions.

- Gaudefroy de Mombyne, T., Mermet, L., 2003. La stratégie d'une ONG internationale d'environnement : articuler biologie et management, action publique et concurrence, Annales des Mines/Gérer E Comprendre, 73, 14-24.

Kalaora, B., Charles, L., 2000. Intervention sociologique et développement durable : le cas de la gestion intégrée des zones côtières, Natures Sciences Sociétés, 8, 2, 31-38.

Lascoumes, P., Setbon, M., 1996. L'Évaluation pluraliste des politiques publiques : enjeux, pratiques, produits, Paris, GAPPCNRS/Commissariat général du plan.

Latour, B., 1999. Politiques de la nature : comment faire entrer les sciences en démocratie, Paris, La Découverte.

$\checkmark$ Leroy, M., 2004. Gestion stratégique des écosystème dans un contexte d'aide internationale : engagements environnementaux et dispositifs de gestion dans la vallée du fleuve Sénégal. Thèse de doctorat, Engref, Paris (à paraître sous forme d'ouvrage aux éditions L'Harmattan).

$\checkmark$ Leroy, M., Mermet, L., 2004. La gestion patrimoniale : innovations et limites de 25 ans de recherche d'une gestion concertée de l'environnement rurale, Séminaire Sciences sociales et Environnement : perspectives franco-allemandes, Strasbourg, 12-14 février.

Margerum, R.D., 1999. Integrated environmental management: the foundations for successful practice, Environmental Management, 24, 2, 151-166.

Margerum, R.D., Born, S.M., 1995. Integrated environmental management: moving from theory to practice, Journal of environmental planning and management, 38, 3, 371-392.

Mermet, L., 1981. Éléments pour une gestion patrimoniale : perspectives, facteurs de blocage, Ministère de l'Environnement Direction de la Prévention des pollutions - Mission des Études statistiques et du Plan/Ministère de l'Urbanisme et du Logement - Mission des Études et de la Recherche Groupe de Prospective/SCORE.

- Mermet, L., 1991. Dans quel sens pouvons-nous gérer l'environnement?, Annales des Mines/Gérer E Comprendre, 22, 68-81.

- Mermet, L., 1992. Stratégies pour la gestion de l'environnement: la nature comme jeu de société?, Paris, L'Harmattan.

- Mermet, L., 1996. Les études d'évaluation entre stratégie et méthodologie : l'exemple des politiques publiques en matière de zones humides, Annales des Mines/Gérer $\mathcal{E}$ Comprendre, 46, 55-64.

- Mermet, L., 1998. L'Analyse stratégique de la gestion environnementale, illustrée par les tribulations d'un noyau de population relictuel d'ours bruns (Ursus Arctos) dans les Pyrénées occidentales françaises, Engref/RGTE.

- Mermet, L., 2001. L'Institution patrimoniale du haut Béarn : gestion intégrée de l'environnement ou réaction anti-environnementale?, Annales des Mines/Responsabilité $\mathcal{E}$ Environnement, 21, 9-21. 
- Mermet, L., à paraître (a). Quand le collectif cosmopolitique, hypostasié en "Institution patrimoniale", devient machine à exclure : le cas de l'ours des Pyrénées, in Lolive, J., Soubeyran, O. (Eds), Émergence des cosmopolitiques et refondation de la pensée aménagiste (colloque du Centre culturel international de Cerisy-la-Salle, 20-27 septembre 2003).

- Mermet, L., à paraître (b). Ouvrir de nouveaux espaces critiques : clarifier, renouveler, «pluraliser» les ancrages normatifs des recherches, in Concertation, décision, environnement : regards croisés, vol. IV, Paris, La Documentation française.

Mermet, L., Barnaud, G., 1999. L'action publique en matière de zones humides : revue, 5 ans après l'évaluation des politiques publiques de 1994, Annales des Mines/Responsabilité $\mathcal{E}$ Environnement, 14, 75-78.

- Mermet, L., Cattan, A., Jubault, P., et al., 1993. Évaluation des politiques publiques en matière de zones humides : rapport de première phase, Paris, AScA/Commissariat général du plan - Instance d'évaluation des politiques publiques.

- Mermet, L., Poux, X., 2000. Recherches et actions publiques à l'interface agriculture-biodiversité : comment déplacer le front du débat ?, Le Courrier de l'environnement de l'INRA, 41, 43-55.

Minzberg, H., 1989. Structure et dynamique des organisations, Paris, Les Éditions Agence d'Arc.

Monnier, E., 1992. Évaluation de l'action des pouvoirs publics, Paris, Economica.

Montgolfier, J. de, Natali, J.-M., 1987. Le Patrimoine du futur, Paris, Economica.

- Narcy, J.-B., 2004. Pour une gestion spatiale de l'eau : comment sortir du tuyau?, Bruxelles, Peter Lang.

\ Narcy, J.-B., Mermet, L., 2003. Nouvelles justifications pour une gestion spatiale de l'eau, Natures Sciences Sociétés, 11, 2, 135-145.

Reçu le 26 mars 2004. Accepté le 3 février 2005.
Ollagnon, H., 1979. Propositions pour une gestion patrimoniale des eaux souterraines : l'expérience de la nappe phréatique $\mathrm{d}$ 'Alsace, Bulletin interministériel pour la rationalisation des choix budgétaires, 36 (mars).

Ollagnon, H., 1987. Une nécessaire rencontre des approches théoriques et pragmatiques de la gestion de la nature : l'audit patrimonial de type système-acteurs, Paris, Ministère de l'AgricultureDAFE.

Ostrom, E., 1990. Governing the Commons. The Evolution of Institutions for Collective Action, Cambridge, Cambridge University Press.

Ostrom, E., Gardner, R., Walker, J., 1994. Rules, Games, E Common Pool Resources, Ann Arbor, The University of Michigan Press.

- Poux, X., 2001. Entreprendre l'évaluation environnementale de la PAC. Responsabilité en environnement, Annales des Mines/Responsabilité E Environnement, 23, 72-85.

- Poux, X., Dubien, I., et al., 1996. État des lieux de la population d'ours des Pyrénées. Stratégie de conservation et de renforcement éventuel. Dimensions naturaliste et socio-économique, AScA/Institution patrimoniale du haut Béarn.

Poux, X., Mermet, L., 1998. Un diagnostic territorial et prospectif comme support de la concertation sur l'environnement, in Gestion des territoires ruraux : connaissances et méthodes pour la décision publique, Actes du colloque CemagrefEngref-ENITAC, Clermont-Ferrand, 27-28 avril 1998, Paris, Cemagref, T 2, 529-548

Riveline, C., 1983. Nouvelles approches des processus de décision (les apports des recherches en gestion), Futuribles, 64-77.

Rosnay, J. de, 1975. Le Macroscope, Paris, Le Seuil.

Walters, C.J., 1986. Adaptive Management of Renewable Resources, New York, McMillan.

Watzlawick, P., Weakland, J.H., Fisch, R., 1977. Changements, paradoxes et psychothérapies, Paris, Le Seuil.

To access this journal online: www.edpsciences.org 